CIRJE-F-1132

\title{
Segregation and Public Spending under Social Identification
}

\author{
Mariko Nakagawa \\ The University of Tokyo \\ Yasuhiro Sato \\ The University of Tokyo \\ Kazuhiro Yamamoto \\ Graduate School of Economics, Osaka University
}

November 2019

CIRJE Discussion Papers can be downloaded without charge from:

http://www.cirje.e.u-tokyo.ac.jp/research/03research02dp.html

Discussion Papers are a series of manuscripts in their draft form. They are not intended for circulation or distribution except as indicated by the author. For that reason Discussion Papers may not be reproduced or distributed without the written consent of the author. 


\title{
Segregation and public spending under social identification*
}

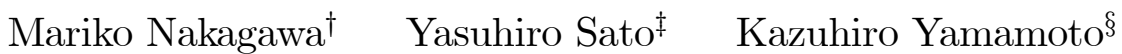

November 19, 2019

\begin{abstract}
We investigate the relationship between segregation and public spending from the viewpoint of theory on social identification by developing a model wherein ethnic minority assimilation and public goods provision are both endogenous. We first show the possibility of multiple equilibria with respect to assimilation: in one equilibrium, individuals belonging to minorities choose to assimilate into the majority society whereas in the other, they reject assimilation, resulting in segregation. We then show that the government's public spending is smaller in the latter equilibrium than in the former one, which is consistent with the empirical finding that segregation decreases public spending. We further examine how changes in the government's objective affect the possibility of multiple equilibria.
\end{abstract}

Keywords: segregation, assimilation, public spending, identification, multiple equilibria JEL classification: H11, H41, J15, Z13

\section{Introduction}

The impacts of ethnic and racial diversity on the economy have attracted much attention in various fields of economics. One strand has focused on the relationship between ethnic or racial fractionalization and public spending. Early contributions including Alesina et al (1999, 2000) provided empirical results showing that regions and countries with higher ethnic diversity and fractionalization have lower public spending. ${ }^{1}$ However, as Gerdes (2011) showed for Denmark,

\footnotetext{
${ }^{*}$ This study was conducted as a part of the project "Spatial Economic Analysis on Intracity Economic Activities and Interregional Economic Activities" undertaken at the Research Institute of Economy, Trade and Industry (RIETI). This work was also supported by JSPS KAKENHI Grant Numbers 17H02519, 18H00841, and $18 \mathrm{H} 00842$.

${ }^{\dagger}$ Center for Spatial Information Science, University of Tokyo, e-mail: mnakagawa@csis.u-tokyo.ac.jp

${ }^{\ddagger}$ Faculty of Economics, University of Tokyo, e-mail: ysato@e.u-tokyo.ac.jp

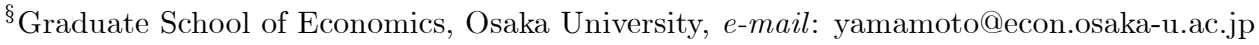

${ }^{1}$ See Alesina and La Ferrara (2005) for a survey on early contributions.
} 
several subsequent studies obtained opposite results. A recent survey by Stichnoth and Van der Straeten (2013) concluded that the results look mixed.

More recent works put more emphasis on the degree of segregation, rather than on the diversity or fractionalization. Alesina and Zhuravskaya (2011) showed that more segregated countries have lower quality governments. Trounstine (2016) argued that "it is not diversity, but segregation along racial lines, that contributes to public goods inequalities across cities" (Trounstine 2016, p.709) and showed that more segregated cities in the United States have lower expenditures on a wide range of public goods. ${ }^{2}$ Moreover, Desmet et al (2017) investigated the empirical relationship between ethnicity and culture, defined as a vector of traits reflecting norms, values, and attitudes, and found that although ethnic and cultural diversity are unrelated in general, in countries where ethnicity is more strongly predictive of culture, public goods provision tends to be lower. This result is consistent with the finding of Trounstine (2016) because ethnic diversity would result in higher cultural diversity if ethnic minorities are segregated than if they are assimilated.

This paper aims to provide a theoretical explanation of the relationship between segregation and public spending from the viewpoint of theory on social identification à la Akerlof (1997) and Akerlof and Kranton $(2000,2010)$. The significance of endogenous identification is also referred to by Alesina and La Ferrara (2005). We utilize a simple framework of identification developed by Shayo (2009) and Sambanis and Shayo (2013) and extend it by introducing public goods provision by a government. We show the existence of multiple equilibria. In one equilibrium, all minority individuals choose to assimilate into (or identify with) the dominant majority group, and in the other, all minority individuals reject the majority's norms. Because segregation is, at least to a certain extent, the result of the minority's decision to assimilate into the majority group, the existence of multiple equilibria can explain why we observe very different levels of segregation for a given degree of ethnic diversity or fractionalization, why there are mixed results regarding the relationship between ethnic fractionalization and public spending, and why ethnic and cultural diversity are unrelated in general as found by Desmet et al (2017). ${ }^{3}$ By examining

\footnotetext{
${ }^{2}$ Note here that even in a highly segregated city or region, if the city or region consists of many small jurisdictions and a particular small jurisdiction is occupied by a particular, homogeneous group of people, then public spending aimed at such a group is shown to increase (La Ferrara and Mele, 2006; Tajima et al., 2018).

${ }^{3}$ We often face the existence of multiple equilibria in this type of model. See for instance, Lindqvist and Östling (2013), Cost Font and Cowell (2015), Holm (2016) and Sato and Zenou (2018). Lindqvist and Östling (2013) analyzed the relationship among ethnic diversity, income inequality, and preference for redistribution. Cost Font and Cowell (2015) provided a survey on the literature of social identity and redistribution preference. Holm (2016) examined the relationship between identification with a nation or a local region and redistribution. Sato and Zenou (2018) investigated how assimilation decision is related to locational segregation.
} 
the multiple equilibria, we show that the share of public spending relative to the total output is higher in the equilibrium in which minority individuals assimilate than in the one in which they reject assimilation. This result is consistent with the findings by Trounstine (2016) and Desmet et al (2017). Moreover, we extend the baseline framework to consider different types of policy determination regimes, and examine under which regime segregation is the most likely to emerge.

Our paper is also related to a growing literature on assimilation of ethnic minorities. Various studies have shown distinct significant influences on the assimilation process for immigrants: the quality of immigrant cohorts (Borjas, 1985), country of origin (e.g., Beenstock et al., 2010; Borjas, 1987, 1992; Chiswick and Miller, 2011), ethnic concentration (e.g., Edin et al., 2003; Lazear, 1999) and personal English skill (e.g., Chiswick and Miller, 1995, 1996; Dustmann and Fabbri, 2003; McManus et al., 1983).

Among this literature, our paper is the most closely related to a significant strand that studies the concept of oppositional cultures among ethnic minorities. In this strand, as in our model, it is possible that ethnic minorities choose to adopt so-called "oppositional" identities, that is, they actively reject (i.e., they are oppositional to) the dominant ethnic behavioral norms. ${ }^{5}$ Of course, it is also possible that they totally assimilate into it. Theoretical works from the viewpoint of cultural identification à la Akerlof (1997) and Akerlof and Kranton (2000, 2010) showed how oppositional identities can emerge as an equilibrium outcome. Such works include Austen-Smith and Fryer (2005), Selod and Zenou (2006), Battu et al. (2007), Bisin et al. (2011a,b, 2016), Panebianco (2014), Carvalho and Koyama (2016), De Marti and Zenou (2017), Eguia (2017), Prummer and Siedlarek (2017), and Verdier and Zenou (2017, 2018). Compared to these existing studies, our contribution is to shed light on the role of a government, and investigate the relationship between assimilation/segregation and the government's behaviors.

This paper is structured as follows. Section 2 presents the baseline framework. In Section 3, we introduce public goods provision into the baseline framework and examine the relationship

\footnotetext{
${ }^{4}$ There is also a strand developing a measure of ethnic identity. Zimmermann et al. (2007), Constant and Zimmermann (2008), Constant et al. (2009) have proposed a new measure of the ethnic identity of migrants by modeling its determinants and explores its explanatory power for various types of their economic performance. They have proposed the ethnosizer, a measure of the intensity of a person's ethnic identity, which is constructed from information on language, culture, societal interaction, history of migration, and ethnic self-identification.

${ }^{5}$ For example, studies in the United States (but also in Europe for ethnic minorities) have found that African American students in poor areas may be ambivalent about learning standard English and performing well at school because this may be regarded as "acting white" and adopting mainstream identities (Ainsworth-Darnell and Downey, 1998; Battu and Zenou, 2010; Fryer and Torelli, 2010; Bisin et al., 2011b; Patacchini and Zenou, 2016).
} 
between assimilation/segregation and public spending. Section 4 provides an extension and Section 5 concludes.

\section{Baseline framework}

Consider a region (or a city) with a continuum of individuals of size 1. Among them a percentage $\mu$ are members of group $m$ and a percentage $1-\mu$ are members of group $n$. We assume that $\mu<1 / 2$, implying that group $m$ is the minority group and group $n$ is the majority group. If we think of ethnicity, then group $m$ is the ethnic minority group while group $n$ corresponds to the native majority group.

Thus, there are two social groups, $m$ and $n$, which are "categories" that individuals learn to recognize while growing up. Each individual is inherently a member of group $m$ or $n$. Following Shayo (2009) and Sambanis and Shayo (2013), we "don't model the cultural and sociological process by which these categories evolved. Rather, we focus on the process of identification with a given set of social groups. (Sambanis and Shayo, 2013, p.301)" Hence we treat these groups as given and focus on the assimilation decision (identification process) of the ethnic minority group $m$, i.e., whether or not they want to assimilate into majority group $n$. Quite naturally, we assume that majority group $n$ is sufficiently large so that they always identify with their own group and we do not deal with their identification decision. In contrast, each minority individual can either choose to identify with her own group $m$ (i.e., rejection of the majority's norms) or with majority group $n$ (i.e., assimilation). In equilibrium, two different groups of ethnic minorities might emerge: those who choose to assimilate into the majority group's identity, referred to as assimilated minorities, and those who choose to reject the majority group's identity, referred to as oppositional minorities.

In the region, the numéraire is produced by using labor only under the constant returns to scale. Letting $y$ denote the productivity of a native individual, we assume that the productivity of an ethnic minority individual is $y_{J}$, where $J$ represents the group she identifies with $(J=$ $m, n)$, and $y_{n}=y>y_{m}=1$. Because of the assumption of constant returns to scale, the productivity equates to the wage income. Thus, an ethnic minority individual can obtain the same wage income, $y$, as a native individual if she assimilates to majority group $n$, whereas she obtains a lower wage income, 1 , if she rejects to assimilate. ${ }^{6}$ This assumption reflects

\footnotetext{
${ }^{6}$ This assumption implies that assimilation decision afffects only the income level and does not affect employment status. However, in reality, employment possibilities migh depend on who they mainly inteact. Zenou (2015) and Sato and Zenou (2015) theoretically examined the relationship between social inteactions and employment. Introducing such a factor will complicate our analysis and is beyond the scope of our paper. Hence,
} 
various social disadvantages ethnic minorities face, including access to good education, language barriers, and different customs. Once they assimilate into group $n$, they learn the majority's norms and resolve the disadvantages. Of course, full removal of disadvantages by assimilation would be an extreme assumption and it would be more realistic to assume that there remains a certain difference even after assimilation. However, in order to clarify the effect of assimilation, we employ this rather extreme assumption. Thus, when identifying with group $J$, an individual receives the wage income of $y_{J}$, which constitutes a part of her utility. Moreover, we assume they obtain utility from public goods consumption, $g$, where public goods provision is financed by income taxation.

We follow Shayo (2009) and Sambanis and Shayo (2013) in specifying three factors of social identity models. The first factor is the social groups or categories that exist in the environment under consideration. The second factor is the perceived distance between each individual and the typical member of her group. The last factor is the relative status of each group in the region. Our model has two social groups; minority group $m$ and majority group $n$. As we have already explained, we assume that members of group $n$ identify with group $n$. Hence, we investigate whether members of group $m$ identify with group $m$ or group $n$.

Each individual is endowed with an attribute $q_{i}$ depending on group $i$ that she is inherently associated with $(i=m, n)$. We assume no heterogeneity in the attributes within each group. Further, we employ a simple specification of the form: $q_{m}=1$ and $q_{n}=0$. The typical attribute, $\bar{q}_{J}$ of each social group (that is relevant to our current analysis, i.e., $J=m, n$ ) is then given by the mean across group members. Because $q_{i}$ is a binary variable, $\bar{q}_{J}$ is given by the share of individuals of group $m$ origin. Letting $\lambda(\in[0,1])$ denote the share of minority people identifying with majority group $n$, we obtain

$$
\bar{q}_{J}=\left\{\begin{array}{cc}
1 & \text { if } J=m \\
\lambda \mu /(\lambda \mu+1-\mu) & \text { if } J=n
\end{array} .\right.
$$

The perceived distance between each individual and identified group $J$ is given by

$$
\begin{aligned}
\ln D_{m J}(\lambda) & =\ln d\left(\left|q_{m}-\bar{q}_{J}\right|\right) \\
\ln D_{n}(\lambda) & =\ln d\left(\left|q_{n}-\bar{q}_{J}\right|\right)
\end{aligned}
$$

where $d(\cdot)$ is an increasing function of its argument $\left(d^{\prime}(\cdot)>0\right)$ and satisfies that $d(0)=1$ and $d(1)=\bar{d}>1$. Here a prime represents differentiation. Hence, (1) is written as

$$
\begin{aligned}
\ln D_{m J}(\lambda) & =\left\{\begin{array}{cc}
0 & \text { if } J=m \\
\ln d((1-\mu) /(\lambda \mu+1-\mu)) & \text { if } J=n
\end{array}\right. \\
\ln D_{n}(\lambda) & =\ln d(\lambda \mu /(\lambda \mu+1-\mu)) .
\end{aligned}
$$

we assume no effect on employment status. 
We assume that it results in the disutility of the form $\delta \ln D_{m J}(\lambda)$ where $\delta$ is a positive constant. The concept of perceived distance and its adoption of the process of identification originate in the literature of categorization in cognitive psychology (Nosofsky 1986, Turner et al. 1987).

Note here that if we assume that each individual is endowed with an additional attribute if she identifies with the majority group, we obtain the same expression as the above one. Suppose that an individual is endowed with $p_{n}=1$ if she identifies with majority group $n$ and $p_{m}=0$ if she identifies with minority group $m$. Then, the perceived distance becomes

$$
D_{m J}(\lambda)=d\left(\alpha\left|p_{J}-\bar{p}_{J}\right|+\left|q_{m}-\bar{q}_{J}\right|\right)
$$

where $\alpha$ is a positive constant. The significance of $\alpha$ represents the way that attention is divided between the different dimensions involved in this identification process. A larger $\alpha$ implies that an individual pays more attention to the attributes of her origin than to the attributes of the identified group. Given our simple structure, we obtain $\bar{p}_{n}=1$ and $\bar{p}_{m}=0$, implying that (3) boils down to (2).

Social identification includes a component related to the status of the identified group as well as to perceptions of similarity to other group members. And the status of the group is determined through comparisons to other groups (Tajfel and Turner 1986). In our framework, we specify the reference group in such a comparison as the region as a whole, and we assume that the utility from the group status is determined by the difference between the average wage income of the group, $\bar{y}_{J}(\lambda)$, and the average wage income of the region as a whole, $\bar{y}(\lambda)$, i.e., $\sigma \ln \left(\bar{y}_{J}(\lambda) / \bar{y}(\lambda)\right)$ for a minority individual and $\sigma \ln (y / \bar{y}(\lambda))$ for a majority individual, where $\sigma$ is a positive constant. Thus, an individual attains higher utility as the group members obtain higher wage income compared to the average level in the city.

In this paper, we assume that the two factors above, that is, the perceived distance and the group status, affect each individual's utility in addition to her private returns, and specify the utility function as

$$
\begin{aligned}
U_{m J}(\lambda) & =\ln \underbrace{\left[(1-\tau) y_{J}+g^{\gamma}\right]}_{\text {private returns }}-\delta \underbrace{\ln D_{m J}(\lambda)}_{\text {perceived distance }}+\sigma \ln \underbrace{\frac{\bar{y}_{J}(\lambda)}{\bar{y}(\lambda)},}_{\text {social status }} \\
U_{n}(\lambda) & =\ln \left[(1-\tau) y+g^{\gamma}\right]-\delta \ln D_{n}(\lambda)+\sigma \ln \frac{y}{\bar{y}(\lambda)},
\end{aligned}
$$

where $U_{m J}(\lambda)$ and $U_{n}(\lambda)$ are the utility for a native majority individual and that for an ethnic minority individual, respectively. $\gamma$ is a positive constant satisfying $0<\gamma<1 . g$ represents the level of public goods provided by a regional government and $\tau$ describes the income tax rate. The first term represents the utility from the private returns, the second term represents the 
disutility from perceived distance between each individual and the identified group, and the last term represents the utility from the status of the identified group.

Because we focus on the assimilation decision by ethnic minorities, only $U_{m J}(\lambda)$ is relevant when considering the assimilation decision. Here, we employ the concept of a Social Identity Equilibrium developed by Shayo (2009). This concept requires that (i) each player's behavior be consistent with her social identity, (ii) social identities be consistent with the social environment, and (iii) the social environment be determined by the players' behaviors. Put differently, it is the Nash equilibrium wherein the strategy includes each player's behavior and identity choice, which in turn, affect players' payoffs. In our current framework, the players are individuals and a regional government, which we will introduce below, and each individual decides only on her identity whereas the regional government decides on income tax and public goods provision. Hence, on the side of the individuals, it is sufficient to check whether their identity decision is consistent with the social environment, which is determined by the government choice. As we will see below, we face three possibilities depending on the individuals' identity choice, and we here define a Social Identity Equilibrium as follows.

\section{Definition (equilibrium)}

(i) An Assimilation Social Identity Equilibrium (ASIE) is when all minority individuals choose to totally assimilate into the majority group, i.e., all choose the identity of group $n$ and $\lambda=1$.

(ii) An Oppositional Social Identity Equilibrium (OSIE) is when all minority individuals totally reject the social norm of the majority group, i.e., all choose the identity of group $m$ and $\lambda=0$.

(iii) A Mixed Social Identity Equilibrium (MSIE) is when a fraction of minority individuals choose to identify themselves with group $m$ while the other fraction chooses to identify themselves with group $n$, i.e., $0<\lambda<1$.

In order to see the individual's identity decisions, suppose temporarily that the government choice $(\tau$ and $g)$ is fixed. For a given $\tau$ and $g$, an individual of group $m$ origin identifies with group $n$ if $U_{m n}(\lambda)>U_{m m}(\lambda)$, identifies with group $m$ if $U_{m m}(\lambda)>U_{m n}(\lambda)$, and is indifferent between identifying with group $m$ and identifying with group $n$ if $U_{m m}(\lambda)=U_{m n}(\lambda)$. From (4), if we define $\Gamma(\lambda ; \tau, g)$ as

$$
\Gamma(\lambda ; \tau, g) \equiv \ln \frac{(1-\tau) y+g^{\gamma}}{1-\tau+g^{\gamma}}-\delta \ln d\left(\frac{1-\mu}{\lambda \mu+1-\mu}\right)+\sigma \ln y
$$


the condition $U_{m n}(\lambda)>U_{m m}(\lambda)$ is rewritten as

$$
\Gamma(\lambda ; \tau, g)>0 .
$$

Then, the above arguments are summarized by the following lemma.

Lemma 1 For given $\tau$ and $g$, an ethnic minority individual identifies with majority group $n$ if $\Gamma(\lambda ; \tau, g)>0$, and she identifies with minority group $m$ if $\Gamma(\lambda ; \tau, g)<0$. She is indifferent between indentifying with either of the two groups if $\Gamma(\lambda ; \tau, g)=0$.

In order to characterize the possibilities of equilibrium, we here derive equilibrium conditions for fixed $\tau$ and $g$. This enables us to better understand the mechanism that yields multiple equilibria. We differentiate $\Gamma(\lambda ; \tau, g)$ with respect to $\lambda$ to obtain $\Gamma^{\prime}(\lambda ; \tau, g)>0 .{ }^{7}$ From this, we can describe $\Gamma(\lambda ; \tau, g)$ and the possibilities of equilibrium as shown in Figure 1.

[Figure 1 around here]

From Figure 1, we readily know that if $\Gamma(0 ; \tau, g)>0$, we obtain $\Gamma(\lambda ; \tau, g)>0, \forall \lambda$ and the unique Assimilation Social Identity Equilibrium If $\Gamma(1 ; \tau, g)<0$, then we obtain $\Gamma(\lambda ; \tau, g)<0, \forall \lambda$ and the unique Oppositional Social Identity Equilibrium. If $\Gamma(0 ; \tau, g)<0$ and $\Gamma(1 ; \tau, g)>0$, we have multiple equilibria, i.e., Assimilation, Oppositional, and Mixed Social Identity Equilibria. Moreover, we impose a stability condition in the sense that a small perturbation yields incentives that restore the economy to the original equilibrium. From the above definitions, the the two former cases yield stable equilibrium. However, in the last case, the MSIE is not stable because $\Gamma^{\prime}(\lambda ; \tau, g)>0$ implying that a small shock gives individuals incentive to change their identity choice so that the economy diverges either to the ASIE or OSIE. Note further that, when $\Gamma(0 ; \tau, g)=0$ or $\Gamma(1 ; \tau, g)=0$, although we have multiple equilibria, only one of them is stable: only the ASIE is stable for the former and only the OSIE is stable for the latter.

The possibilities of equilibrium depend on the relative population size of the minority group compared to the majority group, $\mu$. By differentiating $\Gamma(0 ; \tau, g)$ and $\Gamma(1 ; \tau, g)$ with respect to $\mu$, we know that $\partial \Gamma(0 ; \tau, g) / \partial \mu=0, \partial \Gamma(1 ; \tau, g) / \partial \mu>0$, and $\lim _{\mu \rightarrow 0} \Gamma^{\prime}(\lambda ; \tau, g)=0 .{ }^{8}$ Hence, we know that a sufficiently small size of minority group results in the unique equilibrium. As the size increases, we are likely to observe multiple equilibria. The following proposition summarizes the possibilities of equilibrium.

Proposition 1 Suppose $g$ and $\tau$ are fixed. (a) There exists the unique stable Assimilation Social Identity Equilibrium if $\Gamma(0 ; \tau, g) \geq 0$. (b) There exists the unique stable Oppositional

\footnotetext{
${ }^{7}$ The derivation of $\Gamma^{\prime}(\lambda ; \tau, g)$ is given in Online Appendix A.

${ }^{8}$ The derivations of $\partial \Gamma(0 ; \tau, g) / \partial \mu, \partial \Gamma(1 ; \tau, g) / \partial \mu$, and $\lim _{\mu \rightarrow 0} \Gamma^{\prime}(\lambda ; \tau, g)=0$ are given in Online Appendix A.
} 
Social Identity Equilibrium if $\Gamma(1 ; \tau, g) \leq 0$. (c) There exist multiple stable equilibria if $\Gamma(0 ; \tau, g)<0$ and $\Gamma(1 ; \tau, g)>0$. When $\mu$ is sufficiently small, we have case (a) or (b). As $\mu$ becomes larger, a possibility of case (c) emerges.

As we can see in Shayo (2009), we often observe multiple equilibria in identification decision. This is because of complementarity in the perceived distance: an ethnic minority individual faces a smaller perceived distance when assimilating to majority group $n$ as the other minority individuals assimilate into group $n$, i.e., $\lambda$ becomes larger. Hence, she has small incentive to assimilate when $\lambda$ is small and large incentive to assimilate when $\lambda$ is large, resulting in multiple equilibria. Note that, in our model, the extent of fractionalization is related to the ethnic composition parameter, $\mu$, and the extent of segregation corresponds to the inverse of the assimilation share, $\lambda$, because the more the minority individuals assimilate into the majority group, the less segregated they would be. Based on this reasoning, we can interpret the results of Proposition 1 as follows: Given a certain level of fractionalization, $\mu$, we might observe a very different situation in terms of segregation (i.e., different levels of $\lambda$ ). This also indicates that it is not the degree of fractionalization but the degree of segregation that matters for economic outcomes.

\section{Government and public goods provision}

We now introduce a government into our baseline model and endogenize $\tau$ and $g$ to explore the relationship between assimilation/segregation and public spending. Suppose that there exists a regional government that determines $\tau$ and $g$. Because we have in mind a democratic policy determination process, we employ the median voter hypothesis. This implies that a regional government maximizes the majority individual's utility, $U_{n}(\lambda)$, in (4) by choosing $\tau$ and $g$. We assume that the government's decision and individuals' decision are simultaneous. Hence, the equilibrium concept we use is the Cournot-Nash equilibrium, wherein the government chooses the public good provision, $g$, and income tax rate, $\tau$, optimally for a given Social Identity Equilibrium (for a given $\lambda$ ), and ethnic minority individuals optimally decide whether to assimilate into the majority group for the government's optimal choice.

The budget constraint of the regional government is given by

$$
g=\tau[(1-\lambda) \mu+(\lambda \mu+1-\mu) y] .
$$

Substituting (7) into (4), we obtain the government's objective function, $U_{n}(\lambda)$, that depends only on the income tax rate, $\tau$. Hence, the government chooses $\tau$ to maximize it. The first-order 
condition of the government's maximization yields ${ }^{9}$

$$
\tau(\lambda)=\left(\frac{\gamma \bar{y}(\lambda)^{\gamma}}{y}\right)^{1 /(1-\gamma)}
$$

Plugging (7) and (8) into (5), we obtain

$$
\Gamma(\lambda)=\ln \frac{(1-\tau(\lambda)) y+(\tau(\lambda) \bar{y}(\lambda))^{\gamma}}{1-\tau(\lambda)+(\tau(\lambda) \bar{y}(\lambda))^{\gamma}}-\delta \ln d\left(\frac{1-\mu}{\lambda \mu+1-\mu}\right)+\sigma \ln y .
$$

Because the sign of $\Gamma^{\prime}(\lambda)$ is now ambiguous, we cannot know the uniqueness of the equilibrium. Still, we can obtain sufficient conditions for the multiple equilibria to exist.

Proposition 2 There exist multiple stable equilibria if $\Gamma(0)<0$ and $\Gamma(1)>0$.

Note that we have a possibility that the MSIE is also stable. Note further that the income tax rate represents the share of public spending relative to the regional total output. Combining this with the fact that (8) yields $\tau^{\prime}(\lambda)>0$, which implies that the equilibrium tax rate increases with the equilibrium share of assimilated ethnic minority individuals, $\lambda$, we obtain the following proposition.

Proposition 3 Suppose multiple stable equilibria exist. A regional government spends more intensively on the public goods in equilibrium with a higher share of assimilated ethnic minority individuals.

The stable Assimilation Social Identity Equilibrium has a higher share of public spending relative to the total output than the stable Oppositional Social Identity Equilibrium. Put differently, segregation reduces public spending as empirically shown by Trounstine (2016).

In the stable ASIE, the economy has no income inequality, and hence, the level of public goods provision is determined purely from the viewpoint of the benefits of consuming public goods. However, as $\lambda$ becomes smaller than one, some ethnic minority individuals choose oppositional identities. Because majorities and assimilated minorities have higher income than oppositional minorities, this yields income inequality. Then, public goods provision has a nature of redistribution, which gets stronger as $\lambda$ decreases, i.e., the more minority individuals choose oppositional identities. Low income individuals welcome such a nature whereas high income individuals do not, which gives a regional government maximizing the majority individual's utility an incentive to lower the tax rate.

At the same time, there is also a possibility that a decline in $\lambda$ induces a higher tax rate for the following reason. A decrease in $\lambda$ decreases the tax base $\bar{y}(\lambda)$ and increases the marginal

\footnotetext{
${ }^{9}$ Because $\partial^{2} U_{n}(\lambda ; \tau) / \partial \tau^{2}<0, \partial U_{n}(\lambda ; \tau) /\left.\partial \tau\right|_{\tau=0}>0$, and $\partial U_{n}(\lambda ; \tau) /\left.\partial \tau\right|_{\tau=1}<0, U_{n}(\lambda ; \tau)$ has a unique maximum in terms of $\tau$. See Online Appendix B.
} 
utility of public goods consumption that declines very quickly as $g$ increases for a given tax rate, which gives the government an incentive to raise the tax rate. In our framework, changes in the marginal utility are modest and the effects of redistribution motives dominate the effects of changing marginal utility. Hence, a regional government maximizing the majority individual's utility reduces the public goods provision as $\lambda$ decreases, with the least public goods provision in the stable OSIE.

\subsection{Welfare analysis}

We now investigate the efficiency properties of equilibrium. Because $\lim _{\mu \rightarrow 0} \Gamma^{\prime}(\lambda)=0, \Gamma(\lambda)$ converges to form a flat line along the $\lambda$-axis in the $\lambda-\Gamma$ plane as $\mu$ goes to zero even when $\tau$ and $g$ are endogenous. Then, there is no possibility of multiple equilibria because $\lim _{\mu \rightarrow 0} \Gamma(1)>0$ and $\lim _{\mu \rightarrow 0} \Gamma(0)<0$ cannot hold simultaneously. Note further that the ethnic minority's utility of deviating from the stable ASIE (resp. OSIE) becomes equal to their utility in the stable OSIE (resp. ASIE) if the ethnic minority's population share is sufficiently small, i.e., $\lim _{\mu \rightarrow 0}\left(U_{m m}(1)-U_{m m}(0)\right)=0$ (resp. $\left.\lim _{\mu \rightarrow 0}\left(U_{m n}(0)-U_{m n}(1)\right)=0\right)$. Then, suppose $\lim _{\mu \rightarrow 0} \Gamma(1)=\lim _{\mu \rightarrow 0}\left(U_{m n}(1)-U_{m m}(1)\right)>0$. In such a case, we have the unique stable ASIE, which results in a higher utility of ethnic minority people than in the OSIE because $\lim _{\mu \rightarrow 0}\left(U_{m n}(1)-U_{m m}(0)\right)=\lim _{\mu \rightarrow 0}\left(U_{m n}(1)-U_{m m}(1)\right)>0$. Moreover, we readily know that $\lim _{\mu \rightarrow 0}\left(U_{n}(1)-U_{n}(0)\right)=0$, implying that the majority people are indifferent between the two equilibria. Hence, assimilation decisions in such equilibrium are efficient in the Pareto sense. We can obtain similar results when $\lim _{\mu \rightarrow 0} \Gamma(0)=\lim _{\mu \rightarrow 0}\left(U_{m n}(0)-U_{m m}(0)\right)<0$ holds true.

Proposition 4 Suppose the share of the ethnic minority population is sufficiently small. Then we have either the unique stable Assimilation Social Identity Equilibrium or the stable Oppositional Social Identity Equilibrium. Assimilation decisions in the unique stable equilibrium are efficient in the Pareto sense.

Hence, as long as the share of the ethnic minority population is small, there is no need for governments to intervene in assimilation decisions. Once the share of the ethnic minority population becomes larger, then we might face the possibility of multiple equilibria, and both ethnic minority and majority people face trade-offs. All people obtain higher private returns in the stable ASIE than in the stable OSIE because of higher public good provision in the former. However, the ethnic minority people now obtain disutility from the perceived distance in the former and that from the social status in the latter. Which dominates the other depends on the ethnic minority's preference for private returns, perceived distance, and social status. Also, 
the majority people obtain disutility from the perceived distance in the former and utility from the social status in the latter.

The above arguments induce us to consider a possibility that the economy might be trapped in a Pareto inferior equilibrium in the presence of multiple equilibria when the population share of the minority is sufficiently large. Suppose that the disutility from the perceived distance and utility from social status are not very prominent. Then, when the share of ethnic minority population is sufficiently small, the economy would be in the unique stable ASIE. However, when the share of ethnic minority population is large, the economy would have multiple stable equilibria and we face a possibility that the economy is trapped in the stable OSIE although the stable ASIE is superior to the stable OSIE in the Pareto sense. In such a case, the government can push the equilibrium from the Pareto-inferior one to the Pareto-superior one by using a one-shot intervention such as a temporary reduction in income tax rate.

\section{Extension}

We can extend our baseline model to investigate the impacts of political regimes on ethnic minority's assimilation. To see this, we first show the effect of income tax (and associated public goods provision) on the incentive to assimilate. Substituting the budget constraint (7) into (5), we obtain

$$
\Gamma(\lambda ; \tau)=\ln \frac{(1-\tau) y+(\tau \bar{y}(\lambda))^{\gamma}}{1-\tau+(\tau \bar{y}(\lambda))^{\gamma}}-\delta \ln d\left(\frac{1-\mu}{\lambda \mu+1-\mu}\right)+\sigma \ln y .
$$

Differentiating $\Gamma(\lambda ; \tau)$ with respect to $\tau$, we obtain ${ }^{10}$

$$
\frac{\partial \Gamma(\lambda ; \tau)}{\partial \tau}<0
$$

Hence, we know that a higher income tax rate makes the stable Assimilation Social Identity Equilibrium less likely to emerge, and the stable Oppositional Social Identity Equilibrium more likely to emerge. Having this result in hands, we investigate the impacts of political regimes on the identification decision.

For this purpose, we consider the following two alternative types of governments: a government that maximizes the Benthamite welfare function (i.e., the benevolent government), and a government that considers the total expenditure as well as the welfare (i.e., the Leviathan). These two regimes and the median voter regime are highly popular in the literature of public finance, and it is worth investigating their effects on the likelihood of each type of equilibrium.

We start from the benevolent government. Its objective function is given by

\footnotetext{
${ }^{10}$ See Online Appendix C for derivation.
} 


$$
W_{B}(\lambda)=\mu\left[\lambda U_{m n}(\lambda)+(1-\lambda) U_{m m}(\lambda)\right]+(1-\mu) U_{n}(\lambda)
$$

The subscript $B$ represents the case of the benevolent government. Differentiating $W_{B}(\lambda)$ with respect to $\tau$ and evaluating it at $\tau=\tau(\lambda)$ given by (8), we find

$$
\begin{aligned}
\left.\frac{\partial W_{B}(\lambda)}{\partial \tau}\right|_{\tau=\tau(\lambda)} & =\left.\frac{\partial \mu(1-\lambda) U_{m m}(\lambda)}{\partial \tau}\right|_{\tau=\tau(\lambda)} \\
& =\mu(1-\lambda) \frac{-1+\gamma \tau(\lambda)^{\gamma-1} \bar{y}(\lambda)^{\gamma}}{1-\tau(\lambda)+(\tau(\lambda) \bar{y}(\lambda))^{\gamma}} \\
& =\mu(1-\lambda) \frac{y-1}{1-\tau(\lambda)+(\tau(\lambda) \bar{y}(\lambda))^{\gamma}} \geq 0,
\end{aligned}
$$

where the equality holds true if and only if $\lambda=1$. Hence, letting $\tau_{B}(\lambda)$ denote the optimal tax rate of the benevolent government, we obtain ${ }^{11}$

$$
\begin{array}{ll}
\tau_{B}(\lambda)>\tau(\lambda) & \text { for } \lambda<1, \\
\tau_{B}(\lambda)=\tau(\lambda) & \text { for } \lambda=1 .
\end{array}
$$

Next, we assume the Leviathan government, of which the objective is given by

$$
W_{L}(\lambda)=\beta W_{B}(\lambda)+(1-\beta) \ln (\tau \bar{y}(\lambda)),
$$

where the subscript $L$ represents the case of the Leviathan government. In this case, the government's objective depends on its expenditure as well as on the welfare of individuals. Note here that the expenditure is given by $\tau \bar{y}(\lambda) . \quad \beta$ is a positive constant satisfying that $0<\beta<1$, and it represents the weight on the welfare in the government's objective. Because the expenditure is strictly increasing in the tax rate, we readily know that the optimal tax rate of the Leviathan government, $\tau_{L}(\lambda)$, satisfies $^{12}$

$$
\tau_{L}(\lambda)>\tau_{B}(\lambda), \quad \forall \lambda
$$

From (9), (10), and (11), we obtain the following proposition.

Proposition 5 The stable Assimilation Social Identity Equilibrium is the least likely to emerge under the Leviathan government, and equally likely to emerge under the median voter and under the benevolent government. The stable Oppositional Social Identity Equilibrium is the least likely to emerge under the median voter and the most likely to emerge under the Leviathan government.

\footnotetext{
${ }^{11}$ The existence and uniqueness of $\tau_{B}(\lambda)$ are shown in Online Appendix D.

${ }^{12}$ See Online Appendix D for the formal proof.
} 
Because the public goods provision has the nature of redistribution in the presence of oppositional minorities (i.e., the case of $\lambda>0$ ), the benevolent government has a higher incentive to provide public goods than the median voter government. The Leviathan government has additional incentive to provide public goods in order to expand its expenditure. The strong implication of this result is that the welfare maximizing benevolent government does not necessarily yield the lowest possibility of the OSIE. Rather, the median voter is the least likely to result in it. Hence, the democratic policy determination process is the best among the three regimes considered here in preventing segregation.

\section{Concluding remarks}

This paper theoretically investigated the relationship between ethnic minority assimilation/segregation and public spending. We showed the existence of multiple stable equilibria, which can explain the mixed empirical results regarding ethnic diversity and public spending. We further showed that public spending decreases in a more segregated equilibrium because they inevitably have a nature of redistribution in the presence of income inequality. We finally showed that the democratic policy determination process is the most likely to prevent the economy from falling into the fully segregated equilibrium among political regimes often employed in the literature of public finance.

\section{References}

[1] Ainsworth-Darnell, James W. and Douglas B. Downey, 1998, Assessing the oppositional culture explanation for racial/ethnic differences in school performance, American Sociological Review 63, 536-553.

[2] Alesina, Alberto, Reza Baqir, and William Easterly, 1999, Public Goods and Ethnic Divisions, Quarterly Journal of Economics 114, 1243-1284.

[3] Alesina, Alberto, Edward Glaeser, and Bruce Sacerdote, 2001, Why doesn't the United States have a European-style welfare state?, Brookings Papers on Economic Activity 2, $187-254$.

[4] Alesina, Alberto, and Eliana La Ferrara, 2005, Ethnic diversity and economic performance, Journal of Economic Literature 43, 762-800. 
[5] Alesina, Alberto, and Ekaterina Zhuravskaya, 2011, Segregtion and the quality of government in a cross section of countries, American Economic Review 101, 1872-1911.

[6] Akerlof, George A., 1997, Social distance and social decisions, Econometrica 65, 1005-1027.

[7] Akerlof, George A., and Rachel E. Kranton, 2000, Economics and identity, Quarterly Journal of Economics 115, 715-753.

[8] Akerlof, George A. and Rachel E. Kranton, 2010, Identity Economics: How Our Identities Shape Our Work, Wages, and Well-Being, Princeton: Princeton University Press.

[9] Austen-Smith, David, and Roland D. Fryer, 2005, An economic analysis of 'acting white', Quarterly Journal of Economics 120, 551-583.

[10] Battu, Harminder, McDonald Mwale, and Yves Zenou, 2007, Oppositional identities and the labor market, Journal Population Economics 20, 643-667.

[11] Battu, Harminder, and Yves Zenou, 2010, Oppositional identities and employment for ethnic minorities: Evidence for England, Economic Journal 120, 52-71.

[12] Beenstock, Michael, Barry Chiswick, and Ari Paltiel, 2010, Testing the immigrant assimilation hypothesis with longitudinal data, Review of Economics of the Household 8, 7-27.

[13] Bisin, Alberto, Eleonora Patacchini, Thierry Verdier, and Yves Zenou, 2011a, Formation and persistence of oppositional identities, European Economic Review 55, 1046-1071.

[14] Bisin, Alberto, Eleonora Patacchini, Thierry Verdier, and Yves Zenou, 2011b, Ethnic identity and labor-market outcomes of immigrants in Europe, Economic Policy 26, 57-92.

[15] Bisin, Alberto, Eleonora Patacchini, Thierry Verdier, and Yves Zenou, 2016, Bend it like Beckham. Ethnic identity and integration, European Economic Review 90, 146-164.

[16] Borjas, George J., 1985, Assimilation, changes in cohort quality, and the earnings of immigrants, Journal of Labor Economics 3,463-489.

[17] Borjas, George J., 1987, Self-selection and the earnings of immigrants, American Economic Review 77, 531-553.

[18] Borjas, George J., 1992, Ethnic capital and intergenerational mobility, Quarterly Journal of Economics 107, 123-150.

[19] Carvalho, Jean-Paul, and Mark Koyama, 2016, Jewish emancipation and schism: Economic development and religious change, Journal of Comparative Economics 44, 562-584. 
[20] Chiswick, Barry R., and Paul W. Miller, 1995, The endogeneity between language and earnings: International analyses, Journal of Labor Economics 13, 246-288.

[21] Chiswick, Barry R., and Paul W. Miller, 1996, Ethnic networks and language proficiency among immigrants, Journal of Population Economics 9, 19-35.

[22] Chiswick, Barry R., and Paul W. Miller, 2011, The 'negative' assimilation of immigrants: A special case, Industrial and Labor Relations Review 64, 502-525.

[23] Constant, Amelie, Liliya Gataullina, and Klaus F. Zimmermann, 2009, Ethnosizing immigrants, Journal of Economic Behavior and Organization 69, 274-287.

[24] Constant, Amelie, and Klaus F. Zimmermann, 2008, Measuring ethnic identity and its impact on economic behavior, Journal of the European Economic Association 6, 424-433.

[25] Cost Font, Joan, and Frank Cowell, 2015, Social identity and redistributive preferences: A survey, Journal of Economic Surveys 29, 357-374.

[26] De Marti, Joan, and Yves Zenou, 2017, Segregation in friendship networks, Scandinavian Journal of Economics 119, 656-708.

[27] Desmet, Klaus, Ignacio Ortuño-Ortín, and Romain Wacziarg, 2017, Culture, ethnicity, and diversity, American Economic Review 107, 2479-2513.

[28] Dustmann, Christian, and Francesca Fabbri, 2003, Language proficiency and labour market performance of immigrants in the UK, Economic Journal 113, 695-717.

[29] Edin, Per-Anders, Peter Fredriksson, and Olof Aslund, 2003, Ethnic enclaves and the economic success of immigrants - Evidence from a natural experiment, Quarterly Journal of Economics 118, 329-357.

[30] Eguia, Jon X., 2017, Discrimination and assimilation at school, Journal of Public Economics $156,48-58$.

[31] Fryer, Roland G.Jr., and Paul Torelli, 2010, An empirical analysis of 'acting white', Journal of Public Economics 94, 380-396.

[32] Gerdes, Christer, 2011, The impact of immigration on the size of government: Empirical evidence from Danish municipalities, Scandinavian Journal of Economics 113, 74-92.

[33] Holm, Josha, 2016, A model of redistribution under social identification in heterogeneous federations, Journal of Public Economics 143, 39-48. 
[34] La Ferrara, Eliana, and Angelo Mele, 2006, Racial segregation and public school expenditure, CEPR Discussion Papers 5750.

[35] Lazear, Edward, 1999, Culture and language, Journal of Political Economy 107, S95-S126.

[36] Lindqvist, Erik, and Robert Östling, 2013, Identity and redistribution, Public Choice 155, 469-491.

[37] McManus, Walter, William Gould, and Finis Welch, 1983, Earnings of Hispanic men: The role of English language proficiency, Journal of Labor Economics 1, 101-103.

[38] Nosofsky, Robert M., 1986, Attention, similarity and the identification-categorization relationship, Journal of Experimental Psychology: General 115, 39-57.

[39] Panebianco, Fabrizio, 2014, Socialization networks and the transmission of interethnic attitudes, Journal of Economic Theory 150, 583-610.

[40] Patacchini, Eleonora, and Yves Zenou, 2016, Racial identity and education in social networks, Social Networks 44, 85-94.

[41] Prummer, Anja, Jan-Peter Siedlarek, 2017, Community leaders and the preservation of cultural traits, Journal of Economic Theory 168, 143-176.

[42] Sato, Yasuhiro, and Yves Zenou, 2015, How urbanization affect employment and social interactions, European Economic Review 75, 131-155.

[43] Sato, Yasuhiro, and Yves Zenou, 2018, Assimilation patterns in cities, CEPR Discussion Papers 13364.

[44] Shayo, Moses, 2009, A model of social identity with an application to political economy: Nation, class, and redistribution, American Political Science Review 103, 147-174.

[45] Sambanis, Nicholas, and Moses Shayo, 2013, Social identification and ethnic conflict, American Political Science Review 107, 294-325.

[46] Selod, Harris, and Yves Zenou, 2006, City-structure, job search, and labor discrimination. Theory and policy implications, Economic Journal 116, 1057-1087.

[47] Stichnoth, Holger, and Karine Van der Straeten, 2013, Ethnic diversity, public spending, and individual support for the welfare state: A review of the empirical literature, Journal of Economic Surveys 27, 364-389. 
[48] Tajfel, Henri, and John Turner, 1986, The social identity theory of intergroup behavior, in S. Worchel and W. Austin (eds.), Psychology of Intergroup Relations, Chicago: Nelson Hall.

[49] Tajima, Yuhki, Krislert Samphantharak, and Kai Ostwald, 2018, Ethnic segregation and public goods: Evidence from Indonesia, American Political Science Review 112, 637-653.

[50] Trounstine, Jessica, 2016, Segregation and inequality in public goods, American Journal of Political Science 60, 709-725.

[51] Turner, John, Michael Hogg, Penelope Oakes, Stephen Reicher, and Margaret Wetherell, 1987, Rediscovering the Social Group: A Self-Categorization Theory, Oxford: Blackwell.

[52] Verdier, Thierry, and Yves Zenou, 2017, The role of social networks in cultural assimilation, Journal of Urban Economics 97, 15-39.

[53] Verdier, Thierry, and Yves Zenou, 2018, Cultural leaders and the dynamics of assimilation, Journal of Economic Theory 175, 374-414.

[54] Zenou, Yves, 2015, A dynamic model of weak and strong ties in the labor market, Journal of Labor Economics 33, 891-932.

[55] Zimmermann, Laura, Klaus F. Zimmermann, and Amelie Constant, 2007, Ethnic selfidentification of first-generation immigrants, International Migration Review 41, 769-781. 


\section{Online Appendices (not for publication)}

Appendix A: Derivations of $\Gamma^{\prime}(\lambda ; \tau, g), \partial \Gamma(0 ; \tau, g) / \partial \mu, \partial \Gamma(1 ; \tau, g) / \partial \mu$, and $\lim _{\mu \rightarrow 0} \Gamma^{\prime}(\lambda ; \tau, g)=$ 0.

$\Gamma(\lambda ; \tau, g)$ is given as

$$
\Gamma(\lambda ; \tau, g) \equiv \ln \frac{(1-\tau) y+g^{\gamma}}{1-\tau+g^{\gamma}}-\delta \ln d(P)+\sigma \ln y,
$$

where $P$ is defined as

$$
P \equiv \frac{1-\mu}{\lambda \mu+1-\mu} .
$$

Fix $\tau$ and $g$. By differentiating $\Gamma(\lambda ; \tau, g)$ with respect to $\lambda$, we obtain

$$
\begin{aligned}
\Gamma^{\prime}(\lambda ; \tau, g) & =\frac{\delta \mu(1-\mu) d^{\prime}(P)}{d(P)(\lambda \mu+1-\mu)^{2}}>0, \\
\lim _{\mu \rightarrow 0} \Gamma^{\prime}(\lambda ; \tau, g) & =0 .
\end{aligned}
$$

Moreover, because we know that

$$
\begin{aligned}
\Gamma(0 ; \tau, g) & =\ln \frac{(1-\tau) y+g^{\gamma}}{1-\tau+g^{\gamma}}-\delta \ln \bar{d}+\sigma \ln y, \\
\Gamma(1 ; \tau, g) & =\ln \frac{(1-\tau) y+g^{\gamma}}{1-\tau+g^{\gamma}}-\delta \ln d(1-\mu)+\sigma \ln y,
\end{aligned}
$$

we can obtain

$$
\begin{aligned}
& \frac{\partial \Gamma(0 ; \tau, g)}{\partial \mu}=0 \\
& \frac{\partial \Gamma(1 ; \tau, g)}{\partial \mu}=\frac{\delta d^{\prime}(1-\mu)}{d(1-\mu)}>0 .
\end{aligned}
$$

Appendix B: Derivation of $\tau(\lambda)$ as the unique solution of the maximization of $U_{n}(\lambda ; \tau)$ with respect to $\tau$

First and second partial derivatives of $U_{n}(\lambda ; \tau)$ with respect to $\tau$ are given as

$$
\begin{aligned}
\frac{\partial U_{n}(\lambda ; \tau)}{\partial \tau} & =\frac{-y+\gamma \bar{y}(\lambda)^{\gamma} \tau^{\gamma-1}}{(1-\tau) y+\tau^{\gamma} \bar{y}(\lambda)^{\gamma}}=\frac{-y \tau^{1-\gamma}+\gamma \bar{y}(\lambda)^{\gamma}}{(1-\tau) \tau^{1-\gamma} y+\tau \bar{y}(\lambda)^{\gamma}}, \\
\frac{\partial^{2} U_{n}(\lambda ; \tau)}{\partial \tau^{2}} & =-\frac{\gamma(1-\gamma) \bar{y}(\lambda)^{\gamma} \tau^{\gamma-2}\left[(1-\tau) y+\tau^{\gamma} \bar{y}(\lambda)^{\gamma}\right]+\left[-y+\gamma \bar{y}(\lambda)^{\gamma} \tau^{\gamma-1}\right]^{2}}{(1-\tau) y+\tau^{\gamma} \bar{y}(\lambda)^{\gamma}}<0 .
\end{aligned}
$$

From (12), we obtain

$$
\begin{aligned}
& \left.\frac{\partial U_{n}(\lambda ; \tau)}{\partial \tau}\right|_{\tau=0}=\infty \\
& \left.\frac{\partial U_{n}(\lambda ; \tau)}{\partial \tau}\right|_{\tau=1}=\frac{-y+\gamma \bar{y}(\lambda)^{\gamma}}{\bar{y}(\lambda)^{\gamma}}<0 .
\end{aligned}
$$


Hence, we know that $U_{n}(\lambda ; \tau)$ is a strictly concave function of $\tau$ and has the unique maximum with respect to $\tau$. The solution of the first-order condition

$$
\frac{\partial U_{n}(\lambda ; \tau)}{\partial \tau}=0
$$

yields the unique maximum. By solving (13) for $\tau$, we obtain (8).

\section{Appendix C: Partial derivative of $\Gamma(\lambda ; \tau)$ with respect to $\tau$}

Partial differentiation of $\Gamma(\lambda ; \tau)$ with respect to $\tau$ yields

$$
\frac{\partial \Gamma(\lambda ; \tau)}{\partial \tau}=\frac{(1-y)\left[(\tau \bar{y}(\lambda))^{\gamma}+(1-\tau) \gamma \tau^{\gamma-1} \bar{y}(\lambda)^{\gamma}\right]}{\left[(1-\tau) y+(\tau \bar{y}(\lambda))^{\gamma}\right]\left[1-\tau+(\tau \bar{y}(\lambda))^{\gamma}\right]}
$$

Because $y>1$, we know that

$$
\frac{\partial \Gamma(\lambda ; \tau)}{\partial \tau}<0
$$

Appendix D: Proof of $\tau_{L}(\lambda)>\tau_{B}(\lambda)$

By partially differentiating $W_{B}(\lambda ; \tau)$ with respect to $\tau$, we obtain

$$
\begin{aligned}
\frac{\partial W_{B}(\lambda ; \tau)}{\partial \tau}= & (\mu \lambda+1-\mu) \frac{-y+\gamma \bar{y}(\lambda)^{\gamma} \tau^{\gamma-1}}{(1-\tau) y+\tau^{\gamma} \bar{y}(\lambda)^{\gamma}}+\mu(1-\lambda) \frac{-1+\gamma \bar{y}(\lambda)^{\gamma} \tau^{\gamma-1}}{1-\tau+\tau^{\gamma} \bar{y}(\lambda)^{\gamma}}, \\
\frac{\partial^{2} W_{B}(\lambda ; \tau)}{\partial \tau^{2}}=- & \left\{\frac{\mu \lambda+1-\lambda}{\left[(1-\tau) y+\tau^{\gamma} \bar{y}(\lambda)^{\gamma}\right]^{2}}\left\{\gamma(1-\gamma) \bar{y}(\lambda)^{\gamma} \tau^{\gamma-2}\left[(1-\tau) y+\tau^{\gamma} \bar{y}(\lambda)^{\gamma}\right]+\left[-y+\gamma \bar{y}(\lambda)^{\gamma} \tau^{\gamma-1}\right]^{2}\right\}\right. \\
& \left.\quad+\frac{\mu(1-\lambda)}{\left(1-\tau+\tau^{\gamma} \bar{y}(\lambda)^{\gamma}\right)^{2}}\left[\gamma(1-\gamma) \bar{y}(\lambda)^{\gamma} \tau^{\gamma-2}\left\{1-\tau+\tau^{\gamma} \bar{y}(\lambda)^{\gamma}+\left[-1+\gamma \bar{y}(\lambda)^{\gamma} \tau^{\gamma-1}\right]^{2}\right\}\right]\right\} \\
< & 0, \\
\left.\frac{\partial W_{B}(\lambda ; \tau)}{\partial \tau}\right|_{\tau=0}=\infty & \infty \\
\left.\frac{\partial W_{B}(\lambda ; \tau)}{\partial \tau}\right|_{\tau=1}= & \left(-\bar{y}(\lambda)^{1-\gamma}+\gamma\right)<0 .
\end{aligned}
$$

Hence, $W_{B}(\lambda ; \tau)$ is a strictly concave function of $\tau$ and has the unique maximum, of which solution is written as $\tau_{B}(\lambda) \in(0,1)$. In addition, because $W_{L}(\lambda ; \tau)$ is a linear combination of $W_{B}(\lambda ; \tau)$ and $\ln (\tau \bar{y}(\lambda)), W_{L}(\lambda ; \tau)$ is also a strictly concave function of $\tau$. By evaluating $\partial W_{L}(\lambda ; \tau) / \partial \tau$ at $\tau=\tau_{B}(\lambda)$, we obtain

$$
\begin{aligned}
\left.\frac{\partial W_{L}(\lambda ; \tau)}{\partial \tau}\right|_{\tau=\tau_{B}(\lambda)} & =\left.\beta \frac{\partial W_{B}(\lambda ; \tau)}{\partial \tau}\right|_{\tau=\tau_{B}(\lambda)}+\frac{1-\beta}{\tau_{B}(\lambda)} \\
& =\frac{1-\beta}{\tau_{B}(\lambda)}>0,
\end{aligned}
$$

where the second equality comes from the first-order condition of the maximization of $W_{B}(\lambda ; \tau)$ with respect to $\tau$ (Note that $\tau_{B}(\lambda)$ is the unique solution of $\partial W_{B}(\lambda ; \tau) / \partial \tau=0$ ). Let $\tau_{L}(\lambda)$ denote the solution of the maximization of $W_{L}(\lambda ; \tau)$ with respect to $\tau$. Then, (14) implies that

$$
\tau_{L}(\lambda)>\tau_{B}(\lambda)
$$




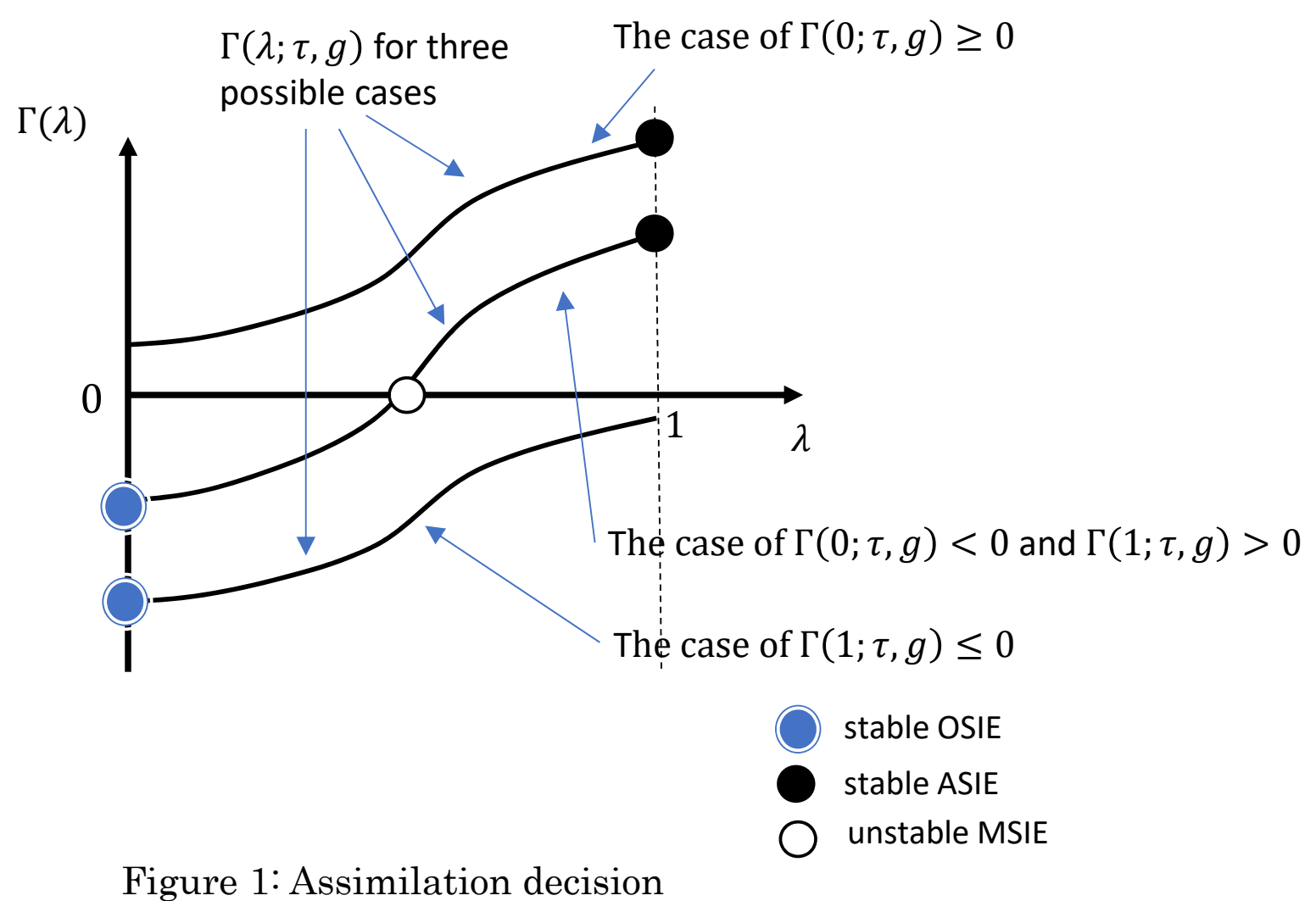

